\title{
КОНКРЕТИЗАЦИЯ ВЫБРАННОГО СМЫСЛА В ПРОЦЕССЕ ВОСПРИЯТИЯ ДВОЙСТВЕННЫХ ИЗОБРАЖЕНИЙ
}

\author{
М.Г. ФИЛИППОВА ${ }^{\text {a }}$ В.М. АЛЛАХВЕРДОВ
}

${ }^{a}$ Санкт-Петербургский государственный университет, 199034, Россия, Санкт-Петербург, Университетская наб., д. 7/9

\begin{abstract}
Резюме
Работа посвящена проверке гипотезы о возрастании конкретизации осознаваемого значения многозначной информации при наличии альтернативных значений, оставшихся неосознанными. Предполагается, что неосознаваемые значения играют важную роль в процессе интерпретации многозначной информации: они ограничивают диапазон смыслов осознаваемого значения за счет отсева тех из них, которые не имеют отношения к выбранному для осознания значению. Конкретизация воспринимаемого объекта происходит путем уточнения контекста восприятия при отвержении менее релевантных альтернатив, которыми этот объект не является. Тем самым устраняется интерференция наиболее подходящему решению. Кроме того, именно неосознаваемые значения обеспечивают постоянство контекста. Конкретизуясь, воспринимаемый объект все менее становится субъективно похож на другие подобные объекты. Мы связываем этот процесс с сужением ассоциативного поля или диапазона эквивалентности значений воспринимаемого объекта. В качестве удобной модели для изучения многозначной информации мы рассматриваем двойственные изображения. В процессе исследования сопоставляется частота нахождения семантических связей слов и изображений в трех условиях: для однозначных изображений, для двойственных изображений при наличии неосознаваемых значений, а также для двойственных изображений при осознании испытуемыми обоих значений. Результаты эксперимента показывают, что условие неосознаваемой многозначности отличается от других условий как по количеству ошибок пропуска семантических связей, так и по способности замечать эти ошибки. Тем самым показано, что наличие неосознаваемых (или негативно выбранных) альтернатив является фактором, способствующим конкретизации осознанного значения. Сделан вывод о том, что устойчивость смысла задается не столько осознанными значениями, сколько негативно выбранными.
\end{abstract}

Ключевые слова: двойственные изображения, неосознаваемые значения, ассоциации, смысл, негативный выбор.

Многозначность - значимый феномен в когнитивной психологии. Практически любая поступающая информация имеет ту или иную степень многозначности, которую человек должен уметь разрешать, не допуская до

Исследование поддержано РФФИ (Проект «Позитивный смысл торможения конкурирующих интерпретаций при восприятии многозначной информации», № 17-06-01014-ОГН). 
осознания нерелевантные контексту альтернативы. Для большинства задач повседневной жизни выбирать единственно правильную интерпретацию поступающей информации - совершенно необходимое умение. Сталкиваясь с двойственной информацией, человек либо интерпретирует ее однозначно и не осознает второго значения либо поочередно осознает то одно, то другое значение. Однако эксперименты показывают, что даже при однозначной интерпретации двойственного стимула второе неосознанное значение какимто образом воспринимается человеком, что проявляется, например, в его ассоциациях, во времени реакции на связанную с неосознанным значением информацию и т.д. (Аллахвердов и др., 2015; Филиппова и др., 2018). Это не позволяет поставить незамеченные значения в один ряд с нейтральными, т.е. непредъявленными. Но если человек реагирует на незамеченное значение, то почему не осознает? Мы предполагаем, что, воспринимая какой-либо объект, человек непременно определяет не только то, чем этот объект является, но и то, чем он не является (см.: Аллахвердов, 2000). В дополнение к другим описанным в психологической литературе механизмам первичной перцептивной обработки информации (например: предвнимание, готовность, селекция, фильтрация, структуризация) мы исходим из предположения о том, что восприятие начинается с неосознаваемого принятия решения о том, что из поступающей информации будет осознано, а что нет (позитивный и негативный выбор). Осознанное, или позитивно выбранное, значение постоянно изменяется. Ведь сознание не способно удерживать неизменную информацию (вспомним У. Джеймса: сознание течет непрерывным потоком, постоянно изменяясь, поэтому, например, невозможно все время думать одну и ту же думу). Однако в процессе восприятия человек должен интерпретировать информацию в определенном и неизменном контексте, а неизменными являются только негативно выбранные значения, поскольку с ними сознание никакой работы не производит. Именно эти отвергнутые сознанием значения обеспечивают постоянство контекста (Аллахвердов, 2000; Allakhverdov et al., 2019).

Есть и другие исследователи, которые признают существование механизма принятия решения о том, что осознавать, а что осознавать не следует. Например, Таль и Бар в своей статье, посвященной размышлениям о судьбе отвергнутых альтернатив восприятия, используя в качестве примера такой предмет, как фен, описывают, по сути, процесс конкретизации значения объекта в процессе восприятия (Tal, Bar, 2014). В первый момент времени, как утверждают авторы, изображение фена будет активировать в памяти разные напоминающие фен объекты (дрель, пистолет, бумеранг и т.д.), которые выступают в качестве гипотез восприятия. Когда в дальнейшем одна из этих гипотез получит поддержку с помощью дополнительной информации, ее активация превысит критический уровень и гипотеза будет осознана. Другие же конкурирующие гипотезы, активированные на первом этапе восприятия, после выбора наиболее релевантной гипотезы будут заблокированы или подавлены. Активация нерелевантных гипотез при этом опустится ниже начального уровня. Такое подавление, согласно Талю и Бару, необходимо только при условии сильно конкурирующих интерпретаций, что они объясняют 
потребностью устранять интерференцию неосознанно выбранному решению. Все подавленные еще до осознания интерпретации они рассматривают в качестве «мешающих активаций». Мы же в подавлении репрезентаций альтернативных значений видим необходимость поддерживать выбранный контекст и конкретизировать выбранное значение. Приведенный пример, на наш взгляд, демонстрирует, каким образом невыбранные альтернативы помогают уточнить контекст восприятия и конкретизировать воспринимаемый объект путем отвержения менее релевантных альтернатив, которыми этот объект не является.

В отличие от теорий селективного внимания, в которых неосознание объясняется ограниченностью ресурсов, необходимостью отсева нерелевантной для текущей деятельности информации и т.д. (Allport et al., 1985; Neill, Valdes, 1992; Tipper, 2001), мы исходим из того, что интерпретация информации связана не только с осознаваемым значением, но и с отвергнутыми (негативно выбранными).

Итак, в процессе неизбежного изменения осознаваемого значения происходит наращивание негативно выбранных значений, объект все больше конкретизируется. Он все меньше становится субъективно похож на другие подобные объекты. Мы связываем этот процесс с сужением ассоциативного поля или диапазона эквивалентности значений воспринимаемого объекта (термин, используемый еще Гарднером - Gardner, 1953). Следствием такого взгляда является предположение о большей конкретизации осознаваемых значений многозначных стимулов при наличии неосознаваемых альтернатив в сравнении со значениями однозначных стимулов. В качестве удобной модели для изучения многозначных стимулов мы рассматриваем двойственные изображения, т.е. такие рисунки, которые позволяют воспринимающему их человеку выбирать, какое из двух значений он осознает. Мы предполагаем, что позитивный выбор одного из значений при восприятии такого рода изображений заведомо провоцирует подавление альтернативных значений, а потому будет обладать более узким диапазоном эквивалентности и меньшим объемом ассоциативного поля, нежели значения однозначных стимулов, предъявляемых вне многозначного контекста. Экспериментальная проверка данного предположения и была основной целью настоящего исследования. Кроме того, мы предполагали возможность и обратного процесса, т.е. расширения диапазона эквивалентности, сопровождающего осознание самого факта многозначности. Это предположение связано с тем, что, когда ассоциации со вторым значением также являются доступными воспринимающему, это может расширить и ассоциативное поле первого значения, в частности, за счет общих для обоих значений ассоциаций.

\section{Метод}

В исследовании приняли участие 27 добровольцев в возрасте от 18 до 32 лет (16 женщин и 11 мужчин). Все они сообщили о том, что имеют нормальное или скорректированное до нормы зрение. 
Проверялась гипотеза, согласно которой осознание только одного из двух значений двойственного изображения сопровождается сужением диапазона эквивалентности этого значения. Двойственными изображениями служили такие рисунки, которые могут быть интерпретированы как принадлежащие одному или другому классу объектов, о чем испытуемым заранее не сообщалось. Помимо двойственных изображений в качестве стимульного материала выступали также однозначные изображения (контрольное условие) и слова (семантически связанные и не связанные с используемыми изображениями). Пример используемого стимульного материала можно видеть в таблицах 1 и 2.

Предполагалось, что наличие неосознаваемой многозначности может повлиять на количество слов, которые испытуемые будут связывать с осознанным ими значением. Операционализация выдвинутой гипотезы состояла в том, что при осознании лишь одного смысла двойственного изображения испытуемые должны выбирать в качестве связанных с этим смыслом меньшее количество слов-ассоциаций, чем в том случае, если смыслы задаются с помощью однозначных изображений. И поскольку в эксперименте испытуемым предлагались уже готовые слова-ассоциации, в качестве зависимой переменной

Таблица 1

Пример двойственных изображений и слов-ассоциаций к ним

\begin{tabular}{|c|c|c|c|}
\hline $\begin{array}{c}\text { Двойственное } \\
\text { изображение }\end{array}$ & $\begin{array}{c}\text { Слова, связанные } \\
\text { с одним значением } \\
\text { изображения }\end{array}$ & $\begin{array}{c}\text { Слова, связанные с } \\
\text { другим значением } \\
\text { изображения }\end{array}$ & $\begin{array}{c}\text { Слова, не } \\
\text { связанные с } \\
\text { изображением }\end{array}$ \\
\hline & осел & морж & дист \\
пони & тюлень \\
телега & море & кратер \\
ношла
\end{tabular}

Таблица 2

Пример однозначных изображений и слов-ассоциаций к ним

\begin{tabular}{|c|c|c|}
\hline Однозначное изображение & $\begin{array}{c}\text { Слова, связанные } \\
\text { с изображением }\end{array}$ & $\begin{array}{c}\text { Слова, не связанные } \\
\text { с изображением }\end{array}$ \\
\hline & $\begin{array}{c}\text { чайник } \\
\text { кипяток } \\
\text { чай } \\
\text { блюдце }\end{array}$ & $\begin{array}{c}\text { картон } \\
\text { асфальт } \\
\text { жук } \\
\text { кольцо }\end{array}$ \\
\hline
\end{tabular}


выступала частота ошибок обнаружения этих ассоциаций. Уделялось внимание ошибкам двух типов: пропуска (если испытуемый не замечал существующей связи изображения и слова) или ложной тревоги (если испытуемый вдруг обнаруживал несуществующую связь изображения и слова). Предполагалось, что сужение диапазона эквивалентности повлечет за собой увеличение ошибок пропуска, а расширение диапазона эквивалентности - возрастание ошибок ложной тревоги. Согласно нашим ожиданиям, восприятие двойственных изображений при наличии неосознаваемых значений сопровождается сужением диапазона эквивалентности, а потому должно повлечь за собой возрастание частоты ошибок пропуска (в сравнении с восприятием однозначных изображений). Кроме того, мы предполагали также возможность расширения диапазона эквивалентности для двойственных изображений, у которых осознаны оба значения, что, согласно нашим ожиданиям, должно сопровождаться возрастанием частоты ошибок ложной тревоги.

Испытуемым предлагалось поочередно выполнять задания двух типов: сначала необходимо было отнести предъявляемое изображение к одному или двум из 7 предлагаемых классов объектов (предметы, транспортные средства, мужчины, женщины, растения, сухопутные животные, водоплавающие животные), а затем - оценить ряд из 16 слов на предмет того, имеют ли они ассоциативную связь с предъявленным ранее изображением. Задача классификации - это прием косвенного определения того, какое из двух значений двойственного изображения (или оба) осознал каждый испытуемый. Этот прием необходим для дальнейшего разделения всех случаев восприятия двойственных изображений на имеющие и не имеющие неосознаваемых значений. Схожая техническая процедура неоднократно использовалась нами ранее (например: Filippova, 2011). Время демонстрации изображений, подлежащих классификации, было ограничено одной секундой. Такое ограничение использовалось для того, чтобы максимально возможным образом снизить вероятность обнаружения многозначности используемых изображений испытуемыми и способствовать формированию у них негативного выбора.

Из 16 относящихся к однозначному изображению слов 8 являлись связанными с ним и 8 - не связанными. Для двойственных изображений использовалась более сложная схема вывода слов-ассоциаций на экран. В случае если испытуемый осознал только одно значение двойственного изображения, ему выводилось 8 слов, связанных с этим значением, 4 слова, связанные с неосознанным значением, а так же 4 не связанных с этим изображением слова. В итоге испытуемый получал также, как и в случае с однозначным изображением, 8 связанных и 8 не связанных с осознанным им значением слов. В случае если испытуемый осознавал оба значения двойственного изображения, ему выводилось 4 слова, связанные с одним из осознанных значений, и 4 слова, связанные со вторым значением, а также 8 несвязанных слов. Изображения предъявлялись испытуемым в одинаковом порядке, но относительно каждого изображения использовался случайный порядок вывода слов. Для оценки наличия ассоциативной связи использовалась задача парадигмы Go/NoGo: oт испытуемого требовалось нажать клавишу при появлении слова, связанного с 
изображением, и проигнорировать нажатие при появлении слова, не связанного с изображением. Время реакции испытуемых было ограничено 1500 мс. Всего в эксперименте использовалось 15 наборов, каждый состоял из одного изображения и 16 слов. 7 из 15 используемых изображений были двойственными, 8 - однозначными (одно из них использовалось в качестве тренировочного задания). После оценки каждого слова испытуемому предоставлялась возможность исправить этот ответ. Испытуемый использовал ее в том случае, если считал, что только что совершил ошибку при оценке связи слова и изображения. Наличие такой возможности позволяло испытуемому перепроверить свое решение, но теперь уже в условиях без жесткого ограничения времени. Нам же это давало дополнительный показатель - вероятность осознания ошибки для проверки гипотезы об устойчивости негативного выбора.

Отбор слов-ассоциаций. Первоначально набор слов-ассоциаций к изображениям подбирался группой экспертов из 10 человек. Из предложенных экспертами слов были отобраны наиболее часто повторившиеся, которые затем были выровнены по количеству букв: для двух значений двойственных изображений подбирались пары слов, состоящие из одинакового количества букв. Затем к каждой паре подбиралось также третье слово, не связанное ни с одним из значений, состоящее из такого же количества букв. По тому же принципу подбирались слова, связанные и не связанные с однозначными изображениями. (Примеры используемых наборов из слов и изображений приведены в таблицах 1 и 2.) Кроме того, была выверена частота встречаемости слов в используемых условиях. Группы слов для ассоциаций к однозначным и двойственным изображениям не имели различий по средней частоте встречаемости $(t=0.88, \mathrm{df}=221, p=0.38)$. Также используемые группы слов (слова-ассоциации к однозначным изображениям, слова-ассоциации к двойственным изображениям и несвязанные слова) не различались по параметрам общего количества употреблений на миллион слов корпуса (ipm: $\mathrm{H}=2.2 ; p=0.821$ ), paвномерности распределения частот в разных частях корпуса (D-Жуйана: $\mathrm{H}=1.45$; $p=0.92)$ и количества сегментов корпуса, в которых встретилось слово ( $\mathrm{R}$ : $\mathrm{H}=1.87 ; p=0.866$ ) (Ляшевская, Шаров, 2009). Данное сравнение осуществлялось с помощью критерия Н Краскела-Уоллиса. Отсутствие различий предполагало эквивалентность созданных наборов слов и позволяло ожидать, что различия в выборе испытуемыми связанных с изображениями слов будут обусловлены экспериментальными условиями.

\section{Результаты}

Несмотря на то что мы стремились ограничить вероятность осознания двойственности предъявляемых изображений испытуемыми, тем не менее в $24 \%$ случаев они все же заметили оба значения двойственных изображений (это можно было оценить по ответам на задание классификации изображений, в котором испытуемым предоставлялась возможность выбрать одновременно два класса объектов). Двойственные изображения были разделены на те, в которых испытуемые осознали только одно значение, и те, в которых осознали 
оба. Сравнивалась частота ошибок пропуска (когда связь слова-ассоциации с осознанным значением изображения не была замечена) и ложной тревоги (когда в качестве ассоциации выбиралось несвязанное слово) для трех условий: для однозначных изображений, для двойственных изображений при наличии неосознаваемых значений, а также для двойственных изображений, в которых были осознаны оба значения. Для сравнения использовался одномерный дисперсионный анализ (Univariate ANOVA). Показатели частоты ошибок в разных условиях представлены в таблице 3.

Частота ошибок пропуска в сравниваемых группах значимо различалась $\left(\mathrm{F}(1,75)=10.2 ; p<0.001 ; \mathrm{R}^{2}=0.22\right)$. Больше всего ошибок пропуска пришлось на двойственные изображения в случае, если испытуемые не осознавали какие-либо их значения. Меньше всего ошибок пропуска происходило в случае с однозначными изображениями. Частота ошибки пропуска в случае, если испытуемый не осознавал двойственности, составила 0.40 , что значимо отличалось от однозначного условия (согласно post hoc критерию LSD, $p<0.001$ ), a также от условия, в котором испытуемый осознавал оба значения двойственного изображения (LSD, $p=0.029)$. В распределении же ошибок ложной тревоги значимых различий обнаружено не было $(\mathrm{F}(1,75)=0.26 ; p=0.77)$.

Далее производился анализ осознания испытуемыми совершенных ошибок пропуска в разных экспериментальных условиях. Как оказалось, испытуемые исправили свои ошибки при пропуске ассоциации к однозначному изображению в 30\% случаев, тогда как к осознанному значению двойственного изображения - в 18\% случаев при отсутствии неосознаваемой многозначности и только в $7 \%$ - при ее наличии $\left(\mathrm{F}(1,75)=6.8 ; p=0.002 ; \mathrm{R}^{2}=0.16\right)$.

\section{Обсуждение}

Полученные результаты показали, что наличие неосознаваемой двойственности изображений сопровождается наибольшим количеством ошибок пропуска слов-ассоциаций к осознанным значениям и затем - наименьшей вероятностью осознания этих ошибок (по сравнению с двумя другими экспериментальными условиями, т.е. как с однозначным условием, так и с условием

Таблица 3

Средняя частота ошибок ложной тревоги и пропуска ассоциаций (и ошибка среднего) для однозначных и двойственных изображений

\begin{tabular}{|c|c|c|c|}
\hline Тип ошибки & $\begin{array}{c}\text { Однозначные } \\
\text { изображения }\end{array}$ & $\begin{array}{c}\text { Двойственные изображе- } \\
\text { ния при наличии неосо- } \\
\text { знаваемых значений }\end{array}$ & $\begin{array}{c}\text { Двойственные изображе- } \\
\text { ния при отсутствии неосо- } \\
\text { знаваемых значений }\end{array}$ \\
\hline $\begin{array}{c}\text { Ошибки } \\
\text { пропуска }\end{array}$ & $0.19(0.030)$ & $0.40(0.029)$ & $0.28(0.032)$ \\
\hline $\begin{array}{c}\text { Ошибки } \\
\text { ложной тревоги }\end{array}$ & $0.15(0.037)$ & $0.14(0.037)$ & $0.11(0.042)$ \\
\hline
\end{tabular}


осознаваемой двойственности). Не подтвердилось предположение о повышении частоты ошибок ложной тревоги при осознании испытуемыми двойственности предъявляемых изображений. Это может быть связано как с тем, что при осознании обоих значений двойственности расширения диапазона эквивалентности и ассоциативного поля каждого из этих значений в реальности не происходит, так и с особенностями организации настоящего эксперимента. Поскольку в данном случае специально не подбирались слова, являющиеся общими ассоциациями для обоих значений, то вопрос о возможности расширения ассоциативного поля одного из значений двойственного изображения при осознании второго может быть адресован будущим исследованиям. По результатам же настоящего эксперимента значениям изображения в условиях осознания двойственности оказался свойствен даже более узкий диапазон эквивалентности, чем в условиях восприятия однозначных изображений, хотя и в меньшей степени, чем в условиях с неосознаваемой двойственностью (т.е. при наличии негативного выбора). В последнем случае наблюдалось наибольшее количество ошибок пропуска семантически связанных словассоциаций, которое показало значимые отличия от обоих контрольных условий. Это свидетельствует о сужении диапазона эквивалентности осознанного значения в условиях неосознаваемой многозначности по сравнению с осознаваемой многозначностью или ее отсутствием. Результаты эксперимента подтверждают нашу гипотезу о том, что негативный выбор способствует конкретизации осознаваемых значений, и также соответствует представлениям об устойчивости негативного выбора, согласно которым негативный выбор защищает принятое решение от опровержений (Аллахвердов, 2000; Allakhverdov, 2008). Негативно выбранные значения, по нашим представлениям, играют важную роль в процессе интерпретации смысла поступающей информации. Позитивно выбранные значения, несомненно, формируют смысл текста. Однако смысл, задаваемый позитивно выбранными значениями, является неустойчивым, быстро меняющимся (течет непрерывным потоком). Негативно выбранные значения обеспечивают постоянство контекста, конкретизируют смысл, уточняя его границы (берега потока), что и приводит к сужению диапазона эквивалентности.

\section{Литература}

Аллахвердов, В. М. (2000). Сознание как парадокс. СПб.: Издательство ДНК.

Аллахвердов, В. М., Гершкович, В. А., Карпинская, В. Ю., Морошкина, Н. В., Науменко, О. В., Тухтиева, Н. Х., Филиппова, М. Г. (2015). Эвристический потенциал концепции Я. А. Пономарева. Психологический журнал, 36(6), 24-34.

Ляшевская, О. Н., Шаров, С. А. (2009). Частотный словарь современного русского языка (на материалах Национального корпуса русского языка). М.: Азбуковник.

Филиппова, М. Г., Костина, Д. И., Мезенцева, М. П. (2018). Динамика узнавания незамеченных значений двойственных изображений и изображений-перевертышей. Психологический журнал, 39(3), 44-56.

Ссылки на зарубежные источники см. в разделе References после англоязычного блока. 
Филиппова Маргарита Георгиевна - научный сотрудник, кафедра общей психологии, Санкт-Петербургский государственный университет, кандидат психологических наук.

Сфера научных интересов: когнитивная и экспериментальная психология, сознание, неосознаваемое восприятие, восприятие многозначной информации.

Контакты: box4fox@yandex.ru

Аллахвердов Виктор Михайлович - профессор, кафедра общей психологии, СанктПетербургский государственный университет, доктор психологических наук, профессор. Сфера научных интересов: когнитивная психология, психология сознания, методология и история психологии, психология искусства.

Контакты: vimiall@gmail.com 


\title{
Concretization of the Chosen Meaning in the Process of Ambiguous Figures Perception
}

\author{
M.G. Filippova ${ }^{a}$, V.M. Allakhverdov ${ }^{a}$ \\ ${ }^{a}$ Saint Petersburg State University, 7/9 Universitetskaya emb., Saint Petersburg, 199034, Russian \\ Federation
}

\begin{abstract}
The paper describes testing the hypothesis of increasing concretization of the consciously perceived meaning of ambiguous information in the presence of alternative meanings that remain unnoticed (i.e. «negatively chosen» meanings). It is assumed that unconscious values play an important role in the process of interpreting ambiguous information: they limit the range of aspects of the consciously perceived meaning by dropping out those that are not related to this meaning. Concretization of the perceived object occurs by thinning the context of perception when rejecting less relevant alternatives, which this object is not. This eliminates the interference with the most suitable decision. In addition, it is the unconscious meaning that provides the constancy of the context. Specifically, the perceived object becomes subjectively less similar to other objects. We link this process with a narrowing of the associative field or the range of equivalence of the meaning of the perceived object. As a convenient model for studying ambiguous information, we consider double meaning figures. In the process of research the frequency of finding the semantic associations of words and figures is compared in three conditions: for unambiguous figures, for ambiguous figures with negatively chosen meanings, and also for ambiguous figures with both meanings consciously perceived by a participant. The results of the experiment show that the condition of unnoticed ambiguity differs from other conditions, both in terms of the number of errors in the omission of semantic associations, and in the ability to notice these errors. Thus, it is shown that the presence of negatively chosen alternatives is a factor contributing to the concretization of a consciously perceived meaning by narrowing its equivalence range. The conclusion is made that the essence is given not so much by conscious meaning, as by negatively chosen ones.
\end{abstract}

Keywords: ambiguous figures, unconscious meanings, associations, meaning, negative choice.

\section{References}

Allakhverdov, V. M. (2000). Soznanie kak paradoks [Consciousness as a paradox]. Saint Petersburg: DNK. (in Russian)

Allakhverdov, V. M. (2008). Awareness as a result of choice. In V. P. Zinchenko \& V. F. Petrenko (Eds.), Psychology in Russia: State of the Art (pp. 136-152). Moscow: MSU/IG-SOTSIN. (in Russian)

Allakhverdov, V. M., Filippova, M. G., Gershkovich, V. A., Karpinskaia, V. Y., Scott, T. V., \& Vladykina, N. P. (2019). Consciousness, learning, and control: On the path to a theory. In A. Cleeremans, 
V. Allakhverdov, \& M. Kuvaldina (Eds.), Implicit learning: 50 years on (pp. 71-107). New York: Routledge.

Allakhverdov, V. M., Gershkovich, V. A., Karpinskaya, V. Y., Moroshkina, N. V., Naumenko, O. V., Tukhtieva, N. H., \& Philippova, M. G. (2015). Heuristic potential of Ya. A. Ponomarev's conception. Psikhologicheskii Zhurnal, 36(6), 24-34. (in Russian)

Allport, D. A., Tipper, S. P., \& Chmiel, N. R. (1985). Perceptual integration and post-categorical filtering. In M. I. Posner \& O. S. Marin (Eds.), Attention and performance XI (pp. 107-132). Hillsdale, NJ: Lawrence Erlbaum Associates, Inc.

Filippova, M. G. (2011). Does unconscious information affect cognitive activity: a study using experimental priming. The Spanish Journal of Psychology, 14(1), 20-36.

Filippova, M. G., Kostina, D. I., \& Mezentseva, M. P. (2018). The recognition dynamics for unnoticed meanings of ambiguous figures. Psikhologicheskii Zhurnal, 39(3), 44-56. (in Russian)

Gardner, R. W. (1953). Cognitive styles in categorizing behavior. Journal of Personality, 22, 214-233.

Lyashevskaya, O. N., \& Sharov, S. A. (2009). Chastotnyi slovar' sovremennogo russkogo yazyka (na materialakh Natsional'nogo korpusa russkogo yazyka) [Frequency dictionary of modern Russian language (based on the materials of National Corpus of Russian language]. Moscow: Azbukovnik. (in Russian)

Neill, W. T., \& Valdes, L. A. (1992). Persistence of negative priming: Steady state or decay? Journal of Experimental Psychology: Learning, Memory, and Cognition, 18(3), 565-576. doi:10.1037/02787393.18.3.565

Tal, A., \& Bar, M. (2014). The proactive brain and the fate of dead hypotheses. Frontiers in Computational Neuroscience, $8,138$.

Tipper, S. P. (2001). Does negative priming reflect inhibitory mechanisms? A review and integration of conflicting views. The Quarterly Journal of Experimental Psychology: Section A, 54(2), 321-343.

Margarita G. Filippova - Research Fellow, Department of General Psychology, Saint Petersburg State University, PhD in Psychology.

Research Area: cognitive and experimental psychology, consciousness, unconscious perception, perception of ambiguous information.

E-mail: box4fox@yandex.ru

Viktor. M. Allakhverdov - Professor, Department of General Psychology, Saint Petersburg State University, DSc in Psychology, Professor.

Research Area: cognitive psychology, psychology of consciousness, methodology and history of psychology, psychology of art.

E-mail: vimiall@gmail.com 